\title{
Auditory system of fruit flies
}

Yuki Ishikawa and Azusa Kamikouchi

Graduate School of Science, Nagoya University, Chikusa, Nagoya, Aichi 464-8602, Japan

*Correspondence to: Azusa Kamikouchi, Graduate School of Science, Nagoya

University, Chikusa, Nagoya, Aichi 464-8602, Japan. Tel. \& fax.: +81 (0)52-789-2903.

E-mail address: kamikouchi@bio.nagoya-u.ac.jp (AK) 


\begin{abstract}
The fruit fly, Drosophila melanogaster, is an invaluable model for auditory research. Advantages of using the fruit fly include its stereotyped behavior in response to a particular sound, and the availability of molecular-genetic tools to manipulate gene expression and cellular activity. Although the receiver type in fruit flies differs from that in mammals, the auditory systems of mammals and fruit flies are strikingly similar with regard to the level of development, transduction mechanism, mechanical amplification, and central projections. These similarities strongly support the use of the fruit fly to study the general principles of acoustic information processing. In this review, we introduce acoustic communication and discuss recent advances in our understanding on hearing in fruit flies.
\end{abstract}

\title{
Keywords
}

Courtship song; Johnston's organ; Antennal ear; Auditory neural circuits; Brain

\section{Introduction}

The courtship of Drosophila melanogaster males is fascinating, involving a series of stereotyped behaviors, each of which requires processing and integration of multiple sensory stimuli, and decision-making (Billeter et al., 2006) (Fig. 1A). During the courtship ritual, the male produces a species-specific courtship song for a female by vibrating his wings (Shorey, 1962; Ewing and Bennet-Clark, 1968). The song is received with the antennal ear (Fig. 1B), whose structure and response properties exhibit no detectable differences between males and females. Intriguingly, many Drosophilid species have their own courtship song characterized by a unique temporal pattern (Ewing and Bennet-Clark, 1968; Cowling and Burnet, 1981). For example, the songs of $D$. melanogaster and its sister species Drosophila simulans have two components, the sine song and the pulse song. In the pulse song, the interval between pulses (interpulse interval, IPI) is species-specific; those of D. melanogaster is $\sim 35 \mathrm{~ms}$ and that of $D$. simulans is $\sim 50$ ms (Moulin et al., 2004). The species-specific pattern of songs, such as the IPI, is widely considered to be important for reproductive isolation in Drosophilid flies. This hypothesis is supported, at least in some species such as D. melanogaster, by observations of an increased mating rate with playback of artificial courtship songs (Bennet-Clark and 
Ewing, 1969; Ritchie et al., 1999). The auditory system of Drosophila thus provides a useful model for understanding how species-specific sounds are represented and appreciated in the brain.

A prominent feature of $D$. melanogaster as a model in neuroscience is that in principle all cells and neurons are genetically identifiable and manipulable at the single-cell level. Thousands of fly strains, each of which expresses transcriptional activators such as GAL4, LexA, and QF in distinct subsets of cell populations (Venken et al., 2011), are readily available from nonprofit stock centers (e.g., Bloomington Stock Center, Drosophila Genetic Resource Center, and Vienna Drosophila Resource Center). By crossing these flies with marker, reporter, or effector strains, any gene of interest can be expressed in specific cell populations. Moreover, the activity of these genes can be turned on and off at will by shifting the temperature or feeding a chemical compound to control the time window of gene expression. This feature provides a great advantage for investigating the general mechanism of sensory processing at the level of molecules, cells, and neural circuits (Simpson, 2009; Kazama, 2014).

The vertebrate and fruit fly auditory systems are strikingly similar with regard to their transduction mechanisms within the auditory organ (Albert et al., 2007), as well as specification of sensory organ precursor cells by the proneural gene atonal at its developmental stage (Jarman et al., 1993; Bermingham et al., 1999). Both the vertebrate cochlea and Drosophila antennal ears have a positive mechanical feedback system within the ear, which actively increases the sensitivity to faint sound (Göpfert et al., 2005). Moreover, noise-induced hearing loss is observed in flies; 1-day exposure to loud noise reduces sound-evoked behavior, sound-evoked nerve potentials, and mitochondrial size in auditory sensory neurons of flies (Christie et al., 2013). Such changes in acoustically traumatized flies are comparable to those in noise-induced hearing loss in mammals (Van Heusden et al., 1981; Böttger and Schacht, 2013), paving the way for using flies in studies of the molecular and genetic mechanisms of hearing loss, in addition to general mechanisms of the auditory system. In this review, we discuss current knowledge on the fly's auditory system by introducing the acoustic behavior, hearing organ, transduction mechanism, and auditory neural circuits of fruit flies. Additional information on the development of auditory organs can be found in Boekhoff-Falk and Eberl (2014). 


\section{Acoustic behavior}

The male courtship song, first described in D. melanogaster (Shorey, 1962), has been reported for more than 110 Drosophila species (Hoikkala, 2005). D. melanogaster male typically produces a courtship sound including sine songs (pure-tone like sound of about $140-\mathrm{Hz}$ frequency) and pulse songs (trains of single cycle pulses with about $35 \mathrm{~ms}$ IPI and 170-Hz intrapulse frequency) (Riabinina et al., 2011). A male typically alternates sine songs with pulse songs, patterned by fast modulations in visual and self-motion signals (Coen et al., 2014). Pulse songs, in particular, have attracted many researchers because flies mate more when played pulse songs with species-typical parameters (Bennet-Clark and Ewing, 1969; Ritchie et al., 1999). This observation indicates that the fruit fly is able to discriminate a particular sound from other sounds. Comparison of the mating rate with/without the courtship song is a classical way of monitoring sound-evoked behavioral changes in fruit flies (Bennet-Clark and Ewing, 1969).

Interestingly, sound-evoked behavioral changes are also observed under a single-sex group condition. Males exposed to pulse songs exhibit increased locomotor activity and begin courting each other if there are no females in the group (Eberl et al., 1997). This acoustically-induced behavior, known as chaining behavior, is not evoked by a pure tone that imitates sine songs (Yoon et al., 2013). Females, on the other hand, exhibit reduced locomotor activity when exposed to pulse songs (Crossley et al., 1995).

These stereotyped behaviors, especially male chaining behavior, are widely used to analyze the behavioral output downstream of hearing (Fig. 2A). The recent development of a computer-based auto detection system for chaining behavior accelerated high-throughput analysis (Yoon et al., 2013). This detection system, termed ChaIN (Chain Index Numerator), scans a movie file and counts the number of flies forming chains on a frame-by-frame basis (Fig. 2B). ChaIN can be used to compare chaining behaviors across Drosophila species and is thus a useful tool for exploring the mechanism of species-specific tuning of the auditory system. A comparison of the behavioral outputs downstream of various artificial songs in D. melanogaster and its sister species $D$. simulans revealed that both species exhibit dramatic selective increases in chaining behavior when exposed to specific auditory cues that correspond to the species-typical pulse songs (Yoon et al., 2013) (Fig. 2C). 


\section{Hearing organ: antennal ear}

As sound has a dual nature, pressure and particle velocity, animals that rely on sound have developed either a pressure receiver or a movement receiver to detect acoustic signals (Ewing, 1989). While the majority of vertebrates and many insect groups (e.g., crickets, grasshoppers, and cicadas) possess a tympanic membrane that moves in response to air pressure fluctuations, some insect groups, such as honeybees, mosquitoes, midges, and fruit flies, use an antennal receiver that tracks the oscillation of air particles (Yack, 2004; Nadrowski et al. 2011). Because the air particle velocity increases more rapidly than pressure when a sound source is approached, the use of particle velocity-sensitive movement receivers is advantageous for close-range communication.

The fruit fly uses antennae to detect particle velocity as near-field sound (Fig. 1B). The arista, a feather-like structure sticking out from the third antennal segment, serves as the sound receiver and vibrates back and forth in response to particle velocity. Movement of the arista is mechanically coupled with the third antennal segment. The basal part of the third antennal segment is called the hook, a structure that articulates the antennal third segment to the second antennal segment. The hook rotates forward and backward as the arista vibrates (Albert and Göpfert, 2015).

Johnston's organ (JO), the largest chordotonal organ in fruit flies, detects sound, gravity, and wind (Kamikouchi et al., 2009; Yorozu et al., 2009). It is located within the second antennal segment and comprises $\sim 200$ chordotonal sensory units called scolopidia, multicellular units that are each comprised of two or three JO neurons, the functional parallel of vertebrate hair cells, and support cells that surround the JO neurons (Brewster and Bodmer, 1995) (Fig. 3A). The scolopidia provide a sealed environment for maintaining and regulating the ionic composition of the endolymph around the cilia of 480 JO neurons (Boekhoff-Falk and Eberl, 2014). The longitudinal axes of scolopidia are radially organized so that their tips are linked to the hook (Fig. 3B, C). Movements of the hook, coupled with the vibration of the arista, stretch and compress the cilia of JO neurons, which in turn activate and inactivate them (Kamikouchi et al 2009). Immobilizing this antennal ear in males abolishes sound-evoked behavior, whereas removing it in females significantly increases the time to mating (Kamikouchi et al., 2009; Vaughan et al., 2014). Together, these observations confirm that the antenna serves as the main hearing organ of fruit flies.

Recently, Mamiya and Dickinson (2015) found that JO neurons also responded 
to the ipsilateral antennal movement during flight. Genetic ablation of JO neurons disrupted slow antiphasic oscillation of the left and right wing stroke amplitudes exhibited by flies flying in the dark. These findings are consistent with a model in which flying flies use JO neurons to detect increases in the wing-induced airflow and that JO neurons are involved in a response that decreases contralateral wing stoke amplitude (Mamiya and Dickinson, 2015). Collectively, JO serves as a multifunctional mechanosensory organ to encode rotations of the third antennal segment relative to the second segment.

\section{Active Amplification}

The estimated intensity range of sound that a female receives from a courting male at a distance of $2.5 \mathrm{~mm}$ is $95 \mathrm{~dB}$ above $50 \mathrm{~nm} / \mathrm{s}$, which corresponds $2.8 \mathrm{~mm} / \mathrm{s}$ (Bennet-Clark, 1971). The sensitivity of the fly's antennal ear far exceeds this intensity. Judging from field potential recordings, JO neurons start responding at particle velocities $5.7 \times 10^{-2}$ $\mathrm{mm} / \mathrm{s}$ for a $300-\mathrm{Hz}$ tone (Lehnert et al., 2013). The active process in the antennal ear of fruit flies enhances the sensitivity, in which a positive mechanical feedback system boosts the antennal vibration and thus actively increases the sound sensitivity of the fly ear (Göpfert and Robert, 2003; Göpfert et al., 2005).

The cellular basis of the active process in the antennal ear has been traced to JO neurons. Mutations that cause structural defects or aberrant intrinsic properties of JO neurons disrupt the proper control of active amplification (Göpfert and Robert, 2003; Göpfert et al., 2005, 2006). In particular, three types of transient receptor potential (TRP) channels expressed in JO neurons play key roles in the regulation of this active process. NompC, also known as TRPN1, is a TRP channel necessary for active amplification (Göpfert and Robert, 2003; Göpfert et al., 2005, 2006); Drosophila NompC is a mechanotransduction channel, the current of which is blocked by FM1-43, a blocker for mechanotransduction channels on hair cells (Yan et al., 2013). Two TRP vanilloid members, Nanchung (Nan) and Inactive (Iav), are also involved in the control of active amplification, by adjusting the amplificatory gain. Nan and Iav form a heteromultimeric channel, which is a selective target of insecticides Pymetrozine and pyrifluquinazon (Nesterov et al., 2015). The excess amplification in nan and iav mutants, which reaches a 10-fold gain in sensitivity, is suppressed in an additional nompC mutation (Göpfert et al., 
2006). Hence, Nan/Iav presumably acts downstream of NompC in the pathway that controls the amplification of the receiver. Although JO neurons express an ortholog of a membrane motor protein Prestin, which contributes to the cochlear amplifier in mammals, this ortholog is dispensable for amplification in Drosophila (Kavlie et al., 2015).

In mammals, the gain of the cochlear amplifier exerted by motile hair cells is centrally controlled by efferent neurons (Warr and Guinan, 1979; Guinan et al., 1983; Warr, 1992; Frolenkov, 2006). Efferent neurons that originate in the olivocochlear complex modulate the electrical and mechanical properties of hair cells, whereby the gain of mechanical amplification is controlled. In contrast, mechanical feedback amplification in Drosophila is not controlled by efferent neurons (Kamikouchi et al., 2010). Silencing the transmission of chemical synapses in all neurons, which disrupts all signaling via chemical synapses to and from JO neurons, does not affect the mechanical feedback gain of the antennal ear. JO itself thus supposedly controls the gain of the amplifier in the fly ear.

\section{Transduction Machinery}

The mechanotransducer channels, which convert sound-induced vibrations into electrical signals, are housed by JO neurons. The short latency of auditory transduction predicts that the transduction complex in JO neurons is directly gated by force, rather than controlled indirectly by a second messenger (Albert et al., 2007; Lehnert et al., 2013). The molecular identity of the mechanotransducer channels in the Drosophila ear is yet to be determined, but the open probability of channels in the absence of a stimulus is estimated to be $\sim 0.5$ (Albert et al., 2007). Stimulus forces alter the open probability of these channels, either in the positive or negative direction. The increase in the open probability is reflected by the nerve responses that are measured in the axons of JO neurons (Albert et al., 2007).

By analogy with vertebrate hair cells, active amplification in Drosophila is thought to depend directly on transduction channel gating (Nadrowski et al., 2008). Accordingly, the NompC and Nan-Iav channels, neuronal TRP channels that play key roles in the control of active amplification, are strong candidate components of the transduction complex that converts the mechanical force imposed on JO neurons into an electrical signal (Effertz et al., 2012; Lehnert et al., 2013). Whereas heteromultimeric $\mathrm{Nan} / \mathrm{Iav}$ channels are localized to the proximal dendrite of the cilia, NompC is localized at 
distal ciliary tips (Gong et al., 2004; Cheng et al., 2010; Lee et al., 2010; Liang et al., 2011). Whereas loss of NompC function does not fully abolish the sound-evoked nerve responses of JO neurons, Nan and Iav are required in both sound-evoked generator currents and sound-evoked nerve responses (Eberl et al., 2000; Effertz et al., 2011, Lehnert et al., 2013). Based on these properties, Nan and Iav seem to be strong candidate components of the transduction complex, but this hypothesis is challenged by the fact that nan and iav mutants retain active amplification of the receiver, which would be abolished if the mechanotransducer channels are lost (Göpfert et al., 2006). Moreover, a recent study demonstrated that neither Nan-Iav heteromers nor their single subunits are activated by hypotonic stimuli, which argues against them being mechanotransduction channels (Nesterov et al., 2015).

Therefore, the molecular mechanism underlying mechanotransduction in the fly ear requires further investigation. Multiple types of mechanotransducer channels may coexist and function in JO neurons of Drosophila (Effertz et al., 2012).

\section{Primary center for auditory sensory neurons}

JO neurons are bipolar mechanosensory neurons, each of which has two neurites, a cilium on one side and an axon on the other side of the cell body (Fig. 3A). Cilia of JO neurons extend toward the hook to sense the movement of the antennal receiver, whereas their axons join the antennal nerve to innervate the brain (Fig. 3B, C). In the brain, the axons spread broadly in the antennal mechanosensory and motor center (AMMC), a pair of neuropil structures lying dorsally to the gnathal ganglia (GNG, also referred to as the subesophageal ganglion; Fig. 4A). A small subset of JO neurons innervates two other brain regions besides the AMMC - the wedge (WED) and GNG.

The projection area of JO neurons has a zonal structure, which comprises five segregated zones, zones A, B, C, D, and E (Kamikouchi et al., 2006) (Fig. 4B, C). Zone A is distributed across three brain regions, the lateral side of the AMMC, the ventral side of the WED, and the dorsal part of the GNG. Zone E is located in the medial side of the AMMC and connects to contralateral zone $\mathrm{E}$ via the commissure of JO neurons (Kamikouchi et al., 2006). Zones B, C, and D are located in the AMMC, between zones A and E. Each JO neuron typically feeds into one zone of the five and thus JO neurons can be classified into five subgroups, subgroups A, B, C, D, and E, according to the zone to 
which they project (Kamikouchi et al., 2006).

The five anatomically classified subgroups of JO neurons are further categorized into vibration-sensitive and deflection-sensitive neurons. Subgroups -A and -B neurons are vibration-sensitive and are activated in a frequency-dependent manner (Kamikouchi et al., 2009; Yorozu et al., 2009; Matsuo et al., 2014); subgroup-B neurons exhibit a frequency preference lower than $100 \mathrm{~Hz}$, while subgroup-A neurons are tuned to higher frequency sound $(>100 \mathrm{~Hz})$. These neurons have important roles in the acoustic response, because disrupting their functions reduces sound-evoked sensitivity and acoustic behaviors (Kamikouchi et al., 2009). On the other hand, subgroups $\mathrm{C}$ and $\mathrm{E}$ are sensitive to static deflection of the antennal receiver to the forward (pull) and backward (push) directions, respectively. These static-deflection subgroups serve as major gravity and wind detectors; silencing or ablating these subgroups abolishes the fly's behavioral response to gravity and wind (Kamikouchi et al., 2009; Yorozu et al., 2009). Subgroup-D neurons are activated by both vibration and deflection stimuli (Matsuo et al., 2014). These five subgroups, each of which has a distinct response property and target zone in the brain, represent information on antennal movement, such as vibrations of various frequencies, static deflections of opposing directions, and a mixture of vibration and deflection, by a combination of activity patterns in the five zones (Fig. 4C).

\section{Auditory pathways in the brain}

Zones A, B, and D, the projection targets of neuronal subgroups that respond to antennal vibrations, together comprise the primary auditory center. Clarification of where and how the information represented in these zones is further processed in the brain is prerequisite to understanding the mechanism of acoustic information processing. To this end, a variety of secondary auditory neurons have been identified anatomically and functionally (Kamikouchi et al., 2009; Lai et al., 2012; Lin et al., 2012; Matsuo et al., 2014; Vaughan et al., 2014).

Many auditory projection neurons, such as AMMC-A1, AMMC-A2, AMMC-B1, and giant fiber (GF) neurons, connect the vibration-sensitive zones A and/or $B$ with the WED, a pair of brain regions located at the dorsal-lateral side of the AMMC. Such an intense connection between the WED and zones A and B predicts that the WED is a major secondary auditory center in the brain. On the other hand, interneurons associated with vibration- and deflection-sensitive zone D so far identified (AMMC-LNs 
and the AMMC-D1 neuron) have no direct connection with the WED (Matsuo et al., 2014). AMMC-LNs are local interneurons that arborize only unilaterally in auditory zones B and D, whereas the AMMC-D1 neuron is a descending neuron that connects zones B, C, D, and E with the thoracico-abdominal ganglia (TAG, also referred to as the ventral nerve chord). Thus, the neural circuit downstream of zone D seemingly forms a distinct pathway from that of zones A and B.

A notable feature of second-order auditory neurons is that most of them project to both sides of the brain. The AMMC-B2 neurons are bilateral local interneurons that connect zone B of both hemispheres. Many other secondary neurons connect the auditory AMMC zones or WED of both hemispheres (Albert and Göpfert, 2015). Connectomics-based analysis of information flow in the Drosophila brain provides a bird's-eye view of the overall connection patterns associated with auditory centers (Shih et al., 2015). This connectome map confirmed the intensive bilateral connections in the auditory neural pathways; when compared with other modalities such as vision and olfaction, intensive bilateral connections are prominent in brain regions that comprise the auditory center. Moreover, the processing core of auditory and other mechanosensory information is predicted from this connectome analysis to be located in the ventromedial protocerebrum, caudalcentral protocerebrum, and dorsomedial protocerebrum of both brain hemispheres. All of these neuropil structures presumably receive input from lower-level auditory centers - the AMMC and caudal ventrolateral protocerebrum (a brain region analogous to the WED). These core brain regions are again characterized by their intensive bilateral connections. Although it is not known whether fruit flies are capable of locating a sound source, such bilateral organization all along the auditory neural pathway, as is observed in mammalian auditory pathways, provides stages of binaural processing in fruit flies. Considering such similarity in the organization of the central auditory pathways, the computational mechanism of binaural acoustic information might be conserved between flies and mammals.

Studies using intracellular recordings and calcium imaging methods revealed response properties of secondary auditory neurons. Four types of secondary auditory neurons, AMMC-A1, AMMC-B1, AMMC-B2, and GF neurons are non-spiking neurons that produce graded potential responses to sound (Tootoonian et al., 2012). AMMC-B1 neurons are functionally subdivided into two types: B1a, which is narrowly tuned for low frequency sound at $\sim 100 \mathrm{~Hz}$, and B1b, which is more broadly tuned (Lai et al., 2012). 
Neuronal-silencing analysis suggest that aPN1 and aLN (al), a type of AMMC-B1 neuron and AMMC-LN, respectively, are necessary for courtship hearing in either male or female flies (Vaughan et al., 2014).

AMMC-A1 neurons, also referred to as giant commissural interneurons, are electrically coupled with GF neurons. Visual looming stimuli elicit spikes in GF, which leads to a short latency jump-flight response through the activation of motor neurons in the thorax (Allen et al., 2006; von Reyn et al., 2014). Although it is not known whether sound information affects this escape behavior, the sound-evoked current from JO neurons propagates to the GF, mainly through gap junctions (Lehnert et al., 2013). The auditory input to electrically coupled AMMC-A1 and GF neurons might affect the activity of the GF system and thus modulate the escape behavior of flies. The GF neuron also responds to deflections of the antenna by an air puff, suggesting that the GF neuron also receives some input from subgroup-E JO neurons that respond to backward static deflection of the antennal ear (Mu et al., 2014).

Third-order auditory neurons have begun to be identified, and one is known to send information from the WED to the ventrolateral protocerebrum (Lai et al., 2012). Further analysis will reveal the organization of entire auditory pathways in the fly brain, and subsequent functional analysis will clarify the neuronal mechanisms that allow flies to interpret the meaning of the sound by discriminating salient acoustic signals from ambient noise.

\section{Perspectives}

Fruit flies have developed a sophisticated acoustic communication system to enhance their mating, together with other sensory modalities, such as vision, olfaction, and gustation. Systematic mapping of auditory pathways in flies will help us to identify the neural substrates that mediate the integration between the auditory system and other sensory systems, as well as the neural circuit mechanisms to process acoustic information. With the power of molecular genetic tools, studies using fruit flies will greatly contribute to the field of auditory research, by elucidating how the brain evaluates sound and integrates it with information from other sensory modalities to effect appropriate behaviors. 


\section{Acknowledgements}

We are very grateful to Daichi Yamada, Dr. Eriko Matsuo, Dr. Hiroshi Ishimoto, and Dr. Nao Morimoto for helpful discussions. Our work described in this review was funded by Decoding and Controlling Brain Informations, PRESTO, Japan Science and Technology Agency, Grant-in-Aids for Young Scientists (A), Scientific Research on Innovate Areas, Challenging Exploratory Research from the Ministry of Education, Culture, Sports, Science and Technology of Japan (Grant Numbers 25710001, 23115717, 25115007, and 25640010), and Human Frontier Science Program (RGY0070/2011).

\section{References}

Albert, J.T., Göpfert, M.C. 2015. Hearing in Drosophila. Curr. Opin. Neurobiol. 34, $79-85$.

Albert, J.T., Nadrowski, B., Göpfert, M.C. 2007. Mechanical signatures of transducer gating in the Drosophila ear. Curr. Biol. 17, 1000-1006.

Allen, M.J., Godenschwege, T.A., Tanouye, M.A., Phelan, P. 2006. Making an escape: Development and function of the Drosophila giant fibre system. Seminars in Cell \& Developmental Biology 17, 31-41.

Bennet-Clark, H.C. 1971. Acoustics of insect song. Nature 234, 255 - 259.

Bennet-Clark, H.C., Ewing, A.W. 1969. Pulse interval as a critical parameter in the courtship song of Drosophila melanogaster. Anim. Behav. 17, 755-759.

Bermingham, N.A., Hassan, B.A., Price, S.D., Vollrath, M.A., Ben-Arie, N., Eatock, R.A., Bellen, H.J., Lysakowski, A., Zoghbi, H.Y. 1999. Math1: an essential gene for the generation of inner ear hair cells. Science 284, 1837-1841.

Billeter, J.-C., Rideout, E.J., Dornan, A.J., Goodwin, S.F. 2006. Control of male sexual behavior in Drosophila by the sex determination pathway. Curr. Biol. 16, R766-R776.

Boekhoff-Falk, G., Eberl, D.F. 2014. The Drosophila auditory system. Wiley Interdiscip.

Rev. Dev. Biol. 3, 179-191.

Böttger, E.C., Schacht, J. 2013. The mitochondrion: a perpetrator of acquired hearing loss. Hearing Res. 303, 12-19.

Brewster, R., Bodmer, R. 1995. Origin and specification of type II sensory neurons in 
Drosophila. Development 121, 2923-2936.

Cheng, L.E., Song, W., Looger, L.L., Jan, L.Y., Jan, Y.N. 2010. The role of the TRP channel NompC in Drosophila larval and adult locomotion. Neuron 67, 373-380.

Christie, K.W., Sivan-Loukianova, E., Smith, W.C., Aldrich, B.T., Schon, M.A., Roy, M., Lear, B.C., Eberl, D.F. 2013. Physiological, anatomical, and behavioral changes after acoustic trauma in Drosophila melanogaster. Proc. Natl. Acad. Sci. U.S.A. 110, 15449-15454.

Coen, P., Clemens, J., Weinstein, A.J., Pacheco, D.A., Deng, Y., Murthy, M. 2014. Dynamic sensory cues shape song structure in Drosophila. Nature 507, 233-237.

Cowling, D.E., Burnet, B. 1981. Courtship songs and genetic control of their acoustic charasteristics in sibling species of the Drosophila melanogaster subgroup. Anim. Behav. 29, 924-935.

Crossley, A.S., Bennet-Clark, H.C., Evert, H.T. 1995. Courtship song components affect male and female Drosophila differently. Anim. Behav. 50, 827-39.

Eberl, D.F., Duyk, G.M., Perrimon, N. 1997. A genetic screen for mutations that disrupt an auditory response in Drosophila melanogaster. Proc. Natl. Acad. Sci. U.S.A. 94, 14837-42.

Eberl, D.F., Hardy, R.W., Kernan, M.J. 2000. Genetically similar transduction mechanisms for touch and hearing in Drosophila. J. Neurosci. 20, 5981-5988.

Effertz, T., Nadrowski, B., Piepenbrock, D., Albert, J.T., Göpfert, M.C. 2012. Direct gating and mechanical integrity of Drosophila auditory transducers require TRPN1. Nat. Neurosci. 15, 1198-1200.

Ewing, A.W. 1989. Arthropod bioacoustics: Neurobiology and behavior, Comstock (Cornell University Press), New York.

Ewing, A.W., Bennet-Clark, H.C. 1968. The courtship songs of Drosophila. Behaviour 31, 288-301.

Frolenkov, G.I. 2006. Regulation of electromotility in the cochlear outer hair cell. J. Physiol. 576, 43-48.

Gong, Z., Son, W., Chung, Y.D., Kim, J., Shin, D.W., McClung, C.A., Lee, Y., Lee, H.W., Chang, D.-J., Kaang, B.-K. 2004. Two interdependent TRPV channel subunits, inactive and Nanchung, mediate hearing in Drosophila. J. Neurosci. 24, 9059-9066.

Göpfert, M., Humphris, A., Albert, J., Robert, D., Hendrich, O. 2005. Power gain 
exhibited by motile mechanosensory neurons in Drosophila ears. Proc. Natl. Acad. Sci. U.S.A. 102, 325-330.

Göpfert, M.C., Robert, D. 2003. Motion generation by Drosophila mechanosensory neurons. Proc. Natl. Acad. Sci. U.S.A. 100, 5514-9.

Göpfert, M.C., Albert, J.T., Nadrowski, B., Kamikouchi, A. 2006. Specification of auditory sensitivity by Drosophila TRP channels. Nat. Neurosci. 9, 999-1000.

Guinan, J.J., Warr, W.B., Norris, B.E. 1983. Differential olivocochlear projections from lateral versus medial zones of the superior olivary complex. J. Comp. Neurol. 221, 358-370.

Hoikkala, A. 2005. Inheritance of male sound characteristics in Drosophila species. In: Drosopoulos, S., Claridge, M.F. (Eds.), Insect sounds and communication: Physiology, behaviour, ecology and evolution. CRC Taylor \& Francis, New York. pp.167-177.

Jarman, A.P., Grau, Y., Jan, L.Y., Jan, Y.N. 1993. atonal is a proneural gene that directs chordotonal organ formation in the Drosophila peripheral nervous system. Cell 73, 1307-1321.

Kamikouchi, A., Shimada, T., Ito, K. 2006. Comprehensive classification of the auditory sensory projections in the brain of the fruit fly Drosophila melanogaster. J. Comp. Neurol. 499, 317-56.

Kamikouchi, A., Albert, J.T., Göpfert, M.C. 2010. Mechanical feedback amplification in Drosophila hearing is independent of synaptic transmission. Eur. J. Neurosci. 31, 697-703.

Kamikouchi, A., Inagaki, H.K., Effertz, T., Hendrich, O., Fiala, A., Göpfert, M.C., Ito, K. 2009. The neural basis of Drosophila gravity-sensing and hearing. Nature 458, 165-71.

Kavlie, R.G., Fritz, J.L., Nies, F., Göpfert, M.C., Oliver, D., Albert, J.T., Eberl, D.F. 2015. Prestin is an anion transporter dispensable for mechanical feedback amplification in Drosophila hearing. J. Comp. Physiol. A 201, 51-60.

Kazama, H. 2014. Systems neuroscience in Drosophila: Conceptual and technical advantages. Neuroscience, doi:10.1016/j.neuroscience.2014.06.035.

Lai, J.S.-Y., Lo, S.-J., Dickson, B.J., Chiang, A.-S. 2012. Auditory circuit in the Drosophila brain. Proc. Natl. Acad. Sci. U.S.A. 109, 2607-2612.

Lee, J., Moon, S., Cha, Y., Chung, Y.D. 2010. Drosophila TRPN (= NOMPC) channel 
localizes to the distal end of mechanosensory cilia. PLoS One 5, e11012.

Lehnert, B.P., Baker, A.E., Gaudry, Q., Chiang, A.S., Wilson, R.I. 2013. Distinct roles of TRP channels in auditory transduction and amplification in Drosophila. Neuron 77, 115-28.

Liang, X., Madrid, J., Saleh, H.S., Howard, J. 2011. NOMPC, a member of the TRP channel family, localizes to the tubular body and distal cilium of Drosophila campaniform and chordotonal receptor cells. Cytoskeleton 68, 1-7.

Lin, S., Kao, C.-F., Yu, H.-H., Huang, Y., Lee, T. 2012. Lineage analysis of Drosophila lateral antennal lobe neurons reveals notch-dependent binary temporal fate decisions. PLoS Biol. 10, e1001425.

Mamiya, A., Dickinson, M.H. 2015. Antennal Mechanosensory Neurons Mediate Wing Motor Reflexes in Flying Drosophila. The Journal of Neuroscience 35, 7977-7991.

Matsuo, E., Yamada, D., Ishikawa, Y., Asai, T., Ishimoto, H., Kamikouchi, A. 2014. Identification of novel vibration-and deflection-sensitive neuronal subgroups in Johnston's organ of the fruit fly. Front. Physiol. 5.

Moulin, B., Aubin, T., Jallon, J.-M. 2004. Why there is a one-way crossability between $D$. melanogaster and D. simulans? Genetica 120, 285-292.

Mu, L., Bacon, J.P., Ito, K., Strausfeld, N.J. 2014. Responses of Drosophila giant descending neurons to visual and mechanical stimuli. J. Exp. Biol. 217, 2121-2129.

Nadrowski, B., Albert, J.T., Göpfert, M.C. 2008. Transducer-based force generation explains active process in Drosophila hearing. Curr. Biol. 18, 1365-1372.

Nesterov, A., Spalthoff, C., Kandasamy, R., Katana, R., Rankl, N.B., Andrés, M., Jähde, P., Dorsch, J.A., Stam, L.F., Braun, F.-J. 2015. TRP channels in insect stretch receptors as insecticide targets. Neuron 86, 665-671.

Riabinina, O., Dai, M., Duke, T., Albert, J.T. 2011. Active process mediates species-specific tuning of Drosophila ears. Curr. Biol. 21, 658-64.

Ritchie, M.G., Halsey, E.J., Gleason, J.M. 1999. Drosophila song as a species-specific mating signal and the behavioural importance of Kyriacou \& Hall cycles in $D$. melanogaster song. Anim. Behav. 58, 649-657.

Shih, C.-T., Sporns, O., Yuan, S.-L., Su, T.-S., Lin, Y.-J., Chuang, C.-C., Wang, T.-Y., Lo, C.-C., Greenspan, R.J., Chiang, A.-S. 2015. Connectomics-based analysis of 
information flow in the Drosophila brain. Curr. Biol. 25, 1249-1258.

Shorey, H. 1962. Nature of the sound produced by Drosophila melanogaster during courtship. Science 137, 677-678.

Simpson, J.H. 2009. Mapping and manipulating neural circuits in the fly brain. Adv. Genet. 65, 79-143.

Tootoonian, S., Coen, P., Kawai, R., Murthy, M. 2012. Neural representations of courtship song in the Drosophila brain. J. Neurosci. 32, 787-98.

Van Heusden, E., Smoorenburg, G. 1981. Eighth-nerve action potentials evoked by tone bursts in cats before and after inducement of an acute noise trauma. Hearing Res. $5,1-23$.

Vaughan, A.G., Zhou, C., Manoli, D.S., Baker, B.S. 2014. Neural pathways for the detection and discrimination of conspecific song in D. melanogaster. Curr. Biol. 24, 1039-1049.

Venken, K.J., Simpson, J.H., Bellen, H.J. 2011. Genetic manipulation of genes and cells in the nervous system of the fruit fly. Neuron 72, 202-230.

von Reyn, C.R., Breads, P., Peek, M.Y., Zheng, G.Z., Williamson, W.R., Yee, A.L., Leonardo, A., Card, G.M. 2014. A spike-timing mechanism for action selection. Nat. Neurosci. 17, 962-970.

Warr, B.W., Guinan, J.J.J. 1979. Efferent innervation of the organ of Corti: two separate systems. Brain Res. 173, 152-155.

Warr, W.B. 1992. Organization of olivocochlear efferent systems in mammals. In: Webster, D.B., Fay, R.R., (Eds.), The mammalian auditory pathway: Neuroanatomy. Springer, New York. pp. 410-448.

Yack, J.E. 2004. The structure and function of auditory chordotonal organs in insects. Microsc. Res. Techniq. 63, 315-37.

Yan, Z., Zhang, W., He, Y., Gorczyca, D., Xiang, Y., Cheng, L.E., Meltzer, S., Jan, L.Y., Jan, Y.N. 2013. Drosophila NOMPC is a mechanotransduction channel subunit for gentle-touch sensation. Nature 493, 221-225.

Yoon, J., Matsuo, E., Yamada, D., Mizuno, H., Morimoto, T., Miyakawa, H., Kinoshita, S., Ishimoto, H., Kamikouchi, A. 2013. Selectivity and plasticity in a sound-evoked male-male interaction in Drosophila. PLoS One 8, e74289.

Yorozu, S., Wong, A., Fischer, B.J., Dankert, H., Kernan, M.J., Kamikouchi, A., Ito, K., Anderson, D.J. 2009. Distinct sensory representations of wind and near-field 
sound in the Drosophila brain. Nature 458, 201-205.

\section{Figure captions}

Fig. 1. Mating behavior and the antenna of Drosophila melanogaster. (A) Behavioral elements in a courtship sequence of Drosophila melanogaster. Males and females are indicated as white and gray colors, respectively. (B) Antennal ear of D. melanogaster.

Fig. 2. Analysis of acoustic behavior in male flies. (A) Detection system of song-induced chaining behavior. Six wing-clipped males are placed in a chamber and exposed to an artificial song. The recorded data are analyzed off-line. (B) ChaIN system. The original data file is subjected to ChaIN, which detects the location and orientation of each fly. When the head of a fly is close enough to the body of another fly, the flies are scored as forming a chain. (C) Antennal ear (Left, top), courtship song (Left, bottom), and acoustic behavior (Right) in Drosophila melanogaster and the sister species D. simulans. The chain index is calculated as the number of individuals forming chains. Lines and ribbons indicate the mean and standard error of the chain index, respectively. Scale bars $=40 \mu \mathrm{m}$ (Left, top) and $0.1 \mathrm{~s}$ (Left, bottom). Modified from Kamikouchi et al., 2009; Yoon et al., 2013, with permission.

Fig. 3 The auditory organ of the fruit fly. (A) The sensory unit in the fruit fly ear. The antennal second segment houses Johnston's organ (Left, location is indicated by the yellow box). The scolopidium of the JO is comprised by the scolopale cell, ligament cell, cap cell, and JO neurons (Right). (B, C) Organization of JO neurons. Frontal (B) and ventral (C) views of three-dimensional reconstructed images are shown. The scolopale cells consist of arrays of thick actin bundles surrounding individual core microtubules and can be visualized with phalloidin (magenta). The nucleus and cytoplasm of JO neurons are visualized with mCD8::GFP (green) and anti-Elav antibody (blue), respectively. Dotted lines in panel $\mathrm{C}$ depict the longitudinal axis of scolopidia. Lines with characters A, D, and L indicate the anterior, dorsal, and lateral, respectively, directions. The bidirectional arrow indicates the direction of the hook's movement. *, the location of the hook. Modified from Kamikouchi et al., 2009, 2013, with permission.

Fig. 4 Target regions of Johnston's organ neurons in the brain. (A) Axonal projections of 
Johnston's organ (JO) neurons in a fruit fly brain. AMMC, antennal mechanosensory and motor center; WED, wedge; GNG, gnathal ganglia; TAG, thoracico-abdominal ganglia. Panels with F (frontal), D (dorsal), and L (lateral), indicate the direction of three-dimensional reconstructed images shown in panel B. (B) Zonal structure of the projection region of JO neurons. The same color-code to label the five zones is used as in panel C. Lines with characters A, D, and L indicate the anterior, dorsal, and lateral, respectively, directions. (C) Five zones as the projection target of JO neurons. Zones A and $\mathrm{B}$ comprise the vibration center, whereas zones $\mathrm{C}$ and $\mathrm{E}$ comprise the deflection center, and zone $\mathrm{D}$ comprises both a vibration and a deflection center. While subgroup-A neurons, which innervate zone A, exhibit a frequency preference higher than $100 \mathrm{~Hz}$, subgroup-B neurons projecting to zone B are tuned to lower frequency sound $(<100 \mathrm{~Hz})$ and subgroup-D neurons and their target zone $\mathrm{D}$ are tuned to middle-range frequency that peaks around 200Hz (Kamikouchi et al., 2009; Yorozu et al., 2009; Matsuo et al., 2014). 
Figure 1

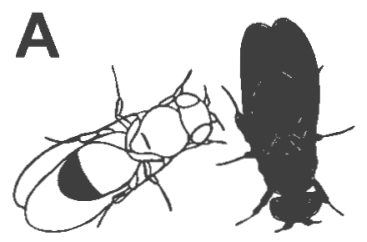

Orientation

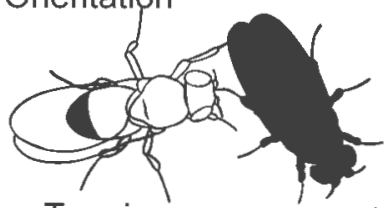

B

Tapping

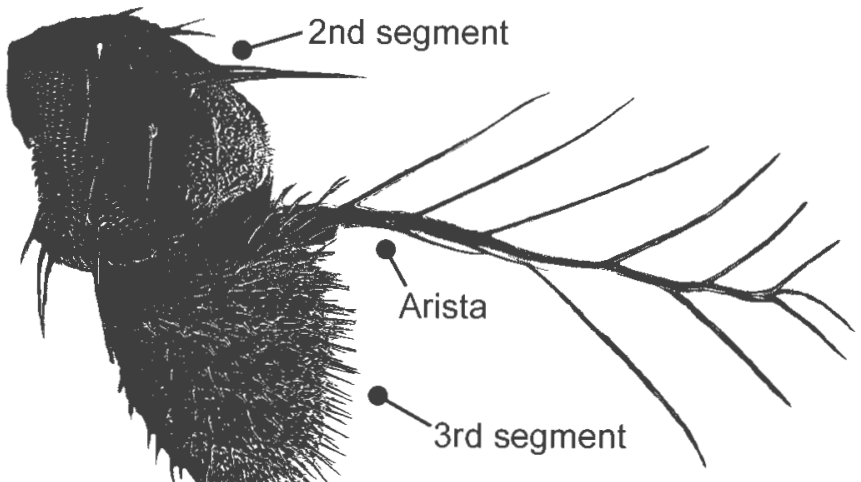

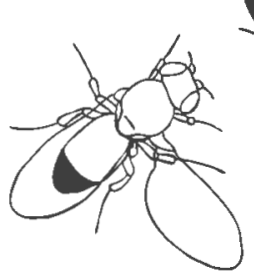

Wing vibration \& Wing display

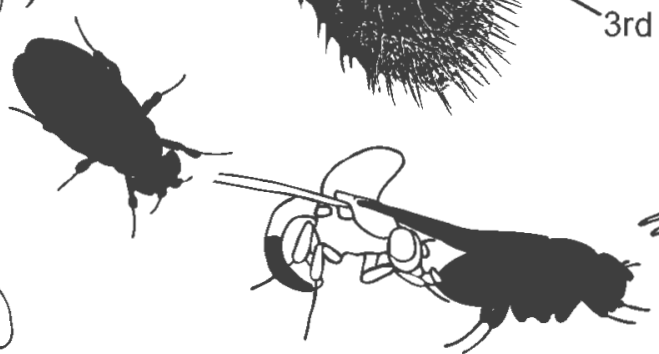

Licking \& Attempting

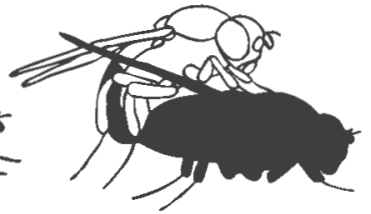

Copulation 


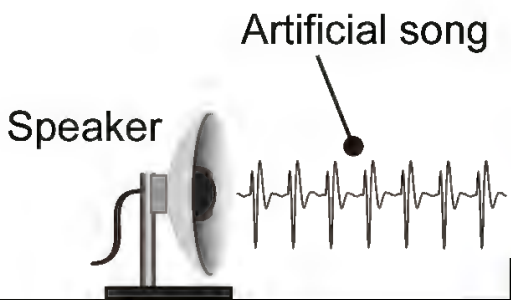

Chamber

Camera

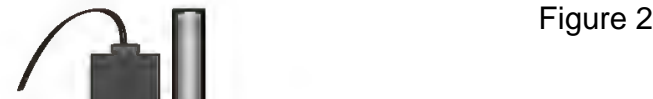

B

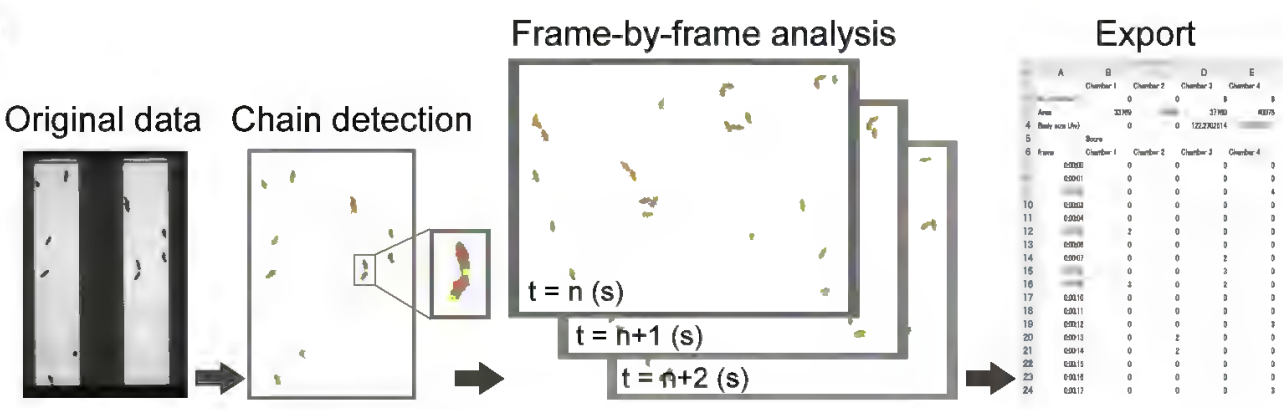

\section{D. melanogaster}

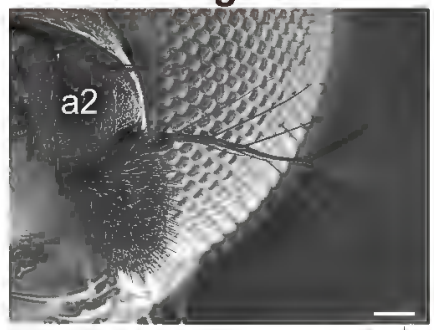

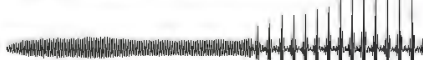

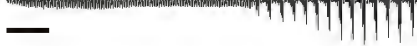

\section{D. simulans}
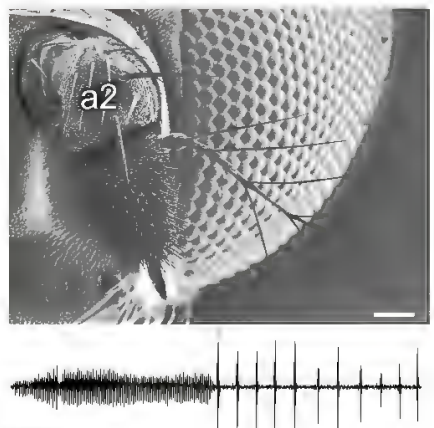
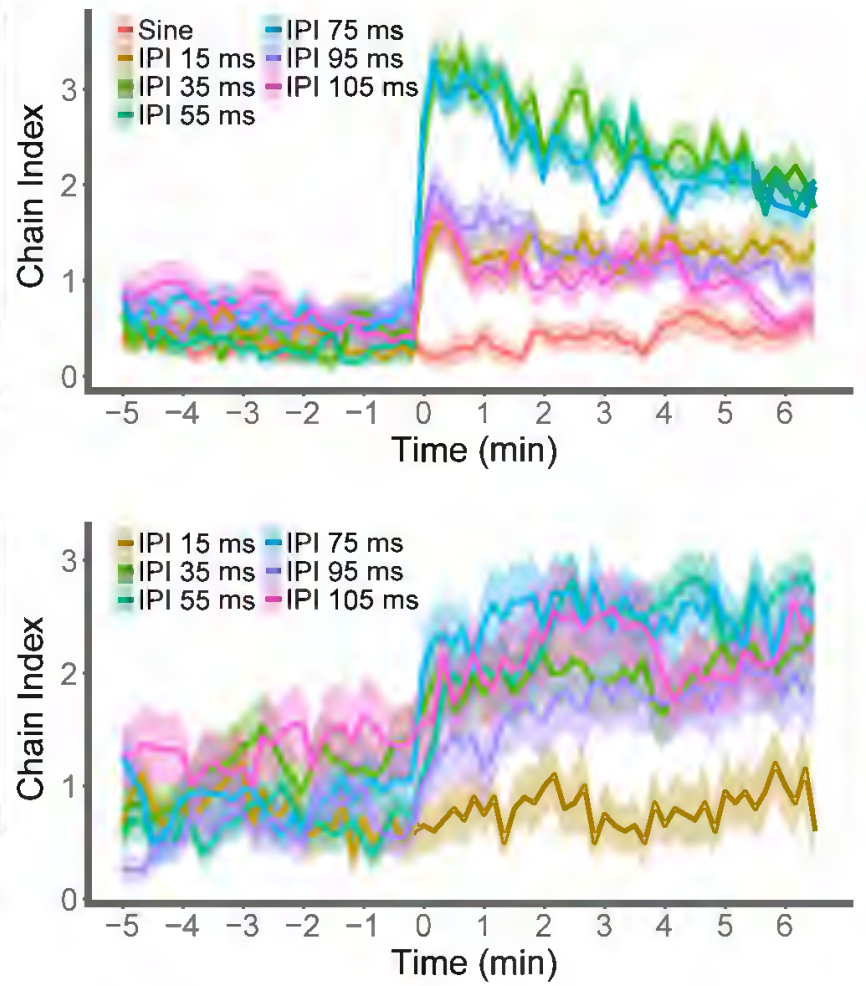
A
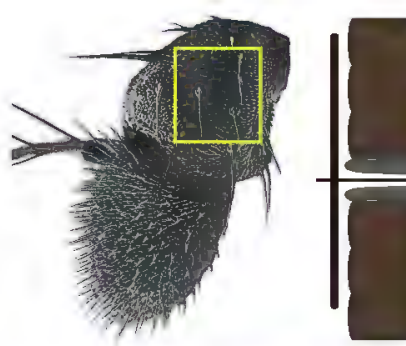

Cap cell Scolopale cell JO neuron
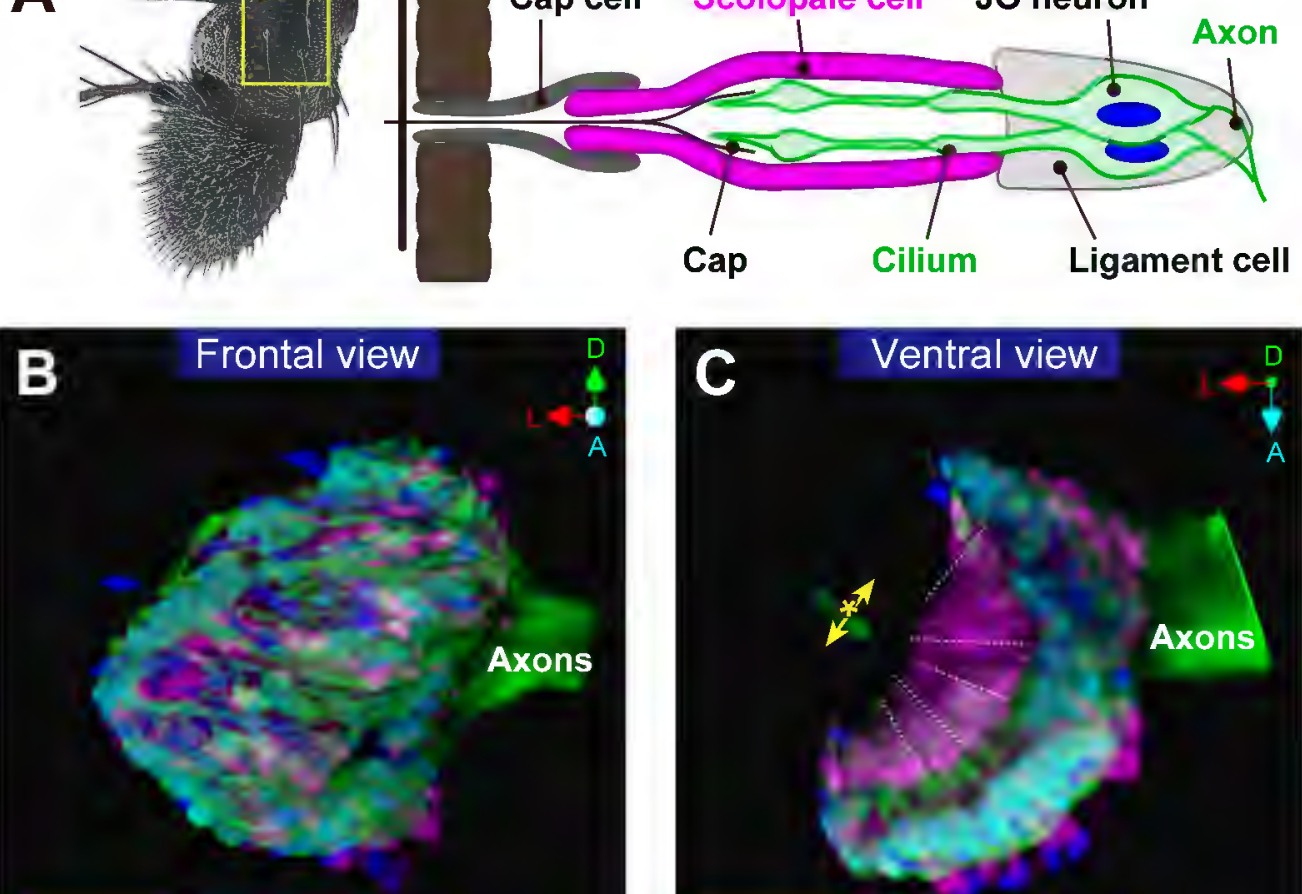

\begin{tabular}{l|ll}
\hline Phalloidin & mCD8::GFP & Elav
\end{tabular}

Phalloidin mCD8::GFP Elav 
C Dorsal view
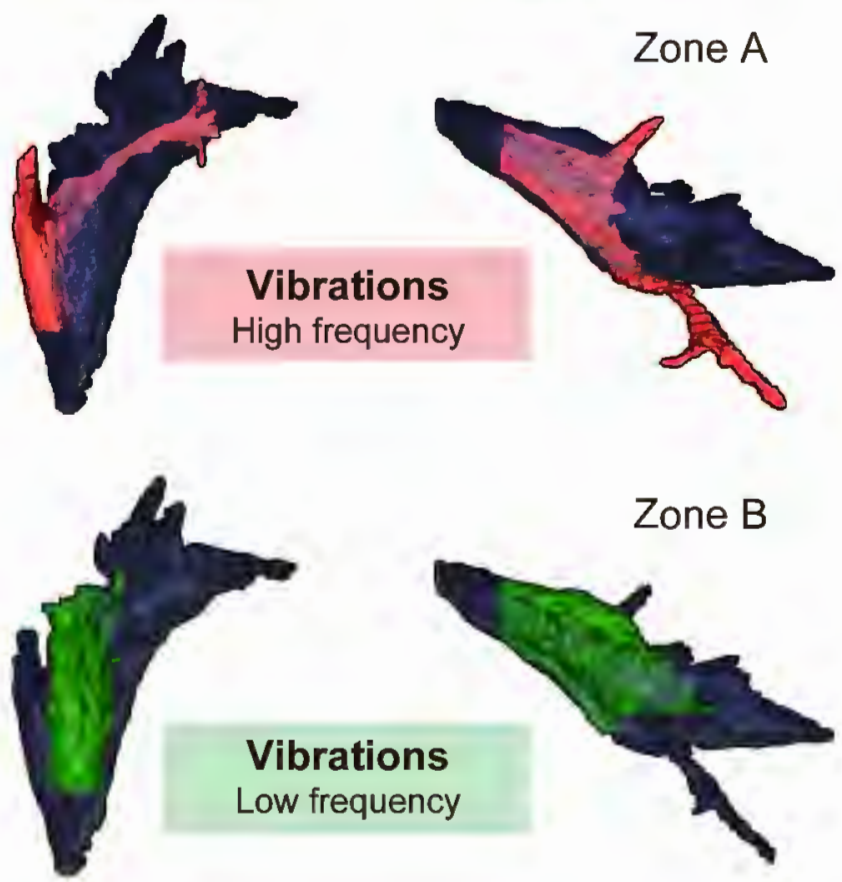

$200 \mu \mathrm{m}$
B

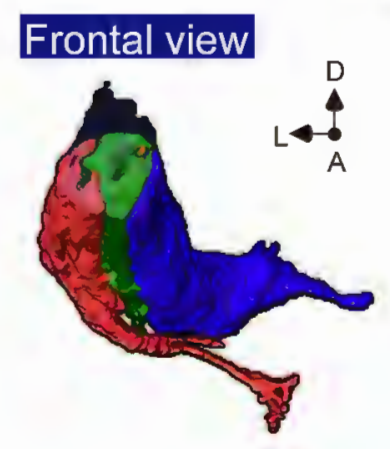

\section{Lateral view}

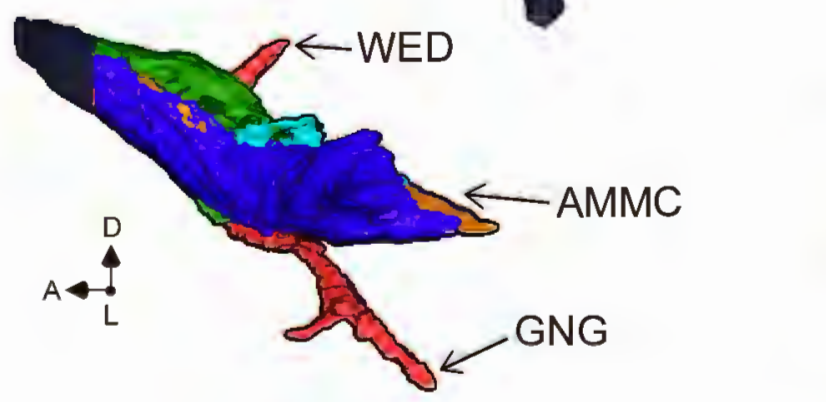

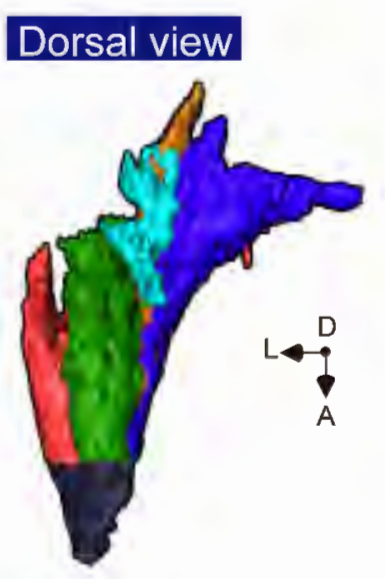

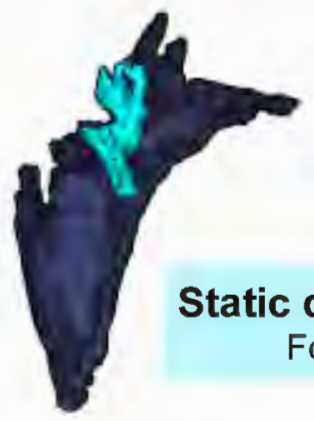

Zone C
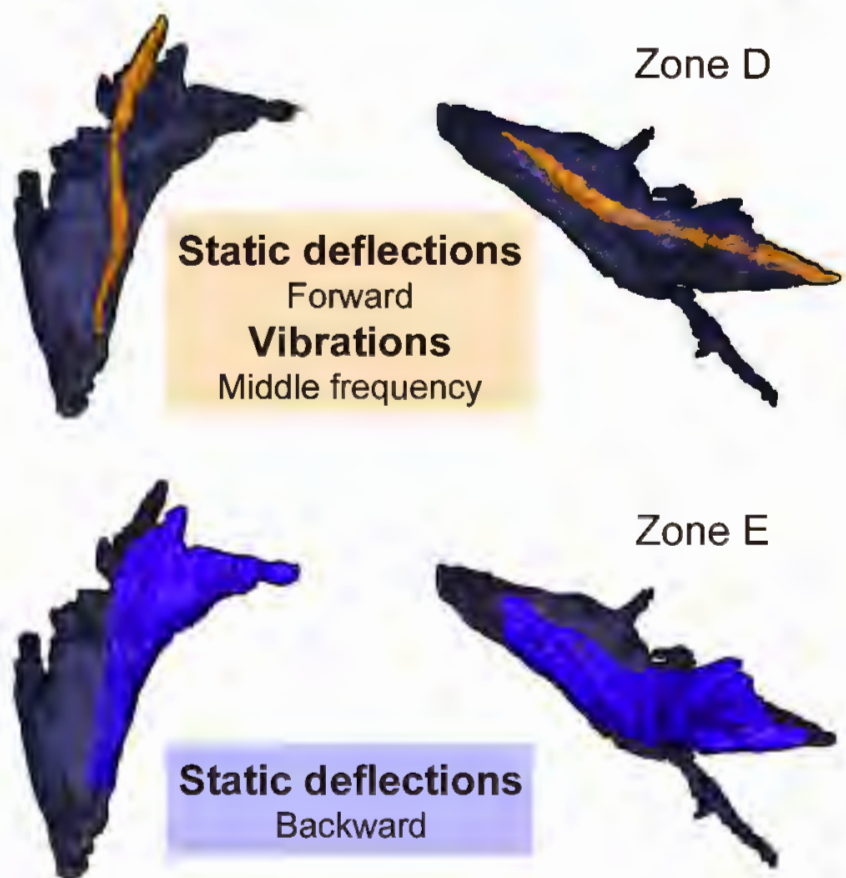